\title{
Anatomy of electric field control of perpendicular magnetic anisotropy at $\mathrm{Fe} / \mathrm{MgO}$ interfaces
}

\author{
F. Ibrahim, H. X. Yang, A. Hallal, B. Dieny, and M. Chshiev \\ Univ. Grenoble Alpes, INAC-SPINTEC, F-38000 Grenoble, France; \\ CNRS, SPINTEC, F-38000 Grenoble, France; \\ and CEA, INAC-SPINTEC, F-38000 Grenoble, France
}

(Received 21 July 2015; revised manuscript received 4 December 2015; published 19 January 2016)

\begin{abstract}
The charge-mediated effect of electric field on the perpendicular magnetic anisotropy (PMA) of Fe/MgO interfaces is investigated using first-principles calculations. We present an approach by discussing this effect in relation to the intrinsic dipole field existing at the $\mathrm{Fe} / \mathrm{MgO}$ interface. A firm correlation between the PMA and the interfacial dipole is established and further verified in the absence of an applied electric field. The on-site projected PMA analysis not only elucidates that the effect of electric field on the PMA extends beyond the interfacial Fe layer, but also shows that the second Fe layer carries the largest contribution to the effect. This observation is interpreted in relation to the orbital hybridization changes induced by applying an electric field.
\end{abstract}

DOI: 10.1103/PhysRevB.93.014429

Manipulating magnetization by the current-induced spintransfer torque (STT) has been extensively studied to conceive magnetic random access memories (MRAMs) where magnetic tunnel junctions (MTJ) serve as prominent devices [1-5]. In particular, out-of-plane magnetized magnetic tunnel junctions (p-MTJ) have attracted considerable interest owing to the strong perpendicular magnetic anisotropy (PMA) which provides both high thermal stability and low switching currents [6-11]. Although remarkable progress has been achieved in this scope, the energy required to write in STT-MRAMs is still rather large (of the order of $100 \mathrm{fJ}$ per write event) but acceptable for a nonvolatile memory. However, this energy could be significantly reduced if the storage layer magnetization switching could be driven by a pulse of electrical field with almost no current actually flowing through the tunnel barrier. The demonstration of electric field control of magnetic properties in ferromagnetic semiconductors [12] and metals [13] has paved the way towards investigating this effect in MTJs where tailoring the PMA is of particular importance to realize fast and low-power-consumption magnetization switching. Several experimental reports have evidenced a strong impact of the electric field on the interfacial PMA in $\mathrm{Fe}(\mathrm{Co}) / \mathrm{MgO}$ systems [14-17]. Theoretical studies have addressed the origin of this effect which was attributed to the spin-dependent screening of the electric field in ferromagnetic metal films [18], and to the change in the relative occupancy of the $3 d$ orbitals of $\mathrm{Fe}$ atoms associated with the accumulation or depletion of electrons at the $\mathrm{Fe} / \mathrm{MgO}$ interface $[19,20]$.

Although electric field control of anisotropy has been intensively investigated in ferromagnet/metal-oxide interfaces, the mechanisms underlying this effect are not yet clear. In fact, the discrepancy between the measured variation of PMA under electric field, which ranges from tenths up to thousands of $\mathrm{fJ} /(\mathrm{V} \mathrm{m})$ in some reports, has suggested alternative mechanisms beyond interfacial charging effects. For instance, it was demonstrated that applying an electric field results in a reversible oxidation or reduction of Fe depending on the voltage polarity [21]. More recently, voltage-driven $\mathrm{O}^{2-}$ migration in a metal/metal-oxide bilayer was observed and electrochemical switching of the interfacial oxidation state was used to completely remove and restore PMA [22].
Another approach that was revealed in a recent experiment discusses the interplay between the electric field controlled PMA and strain properties, showing that an applied electric field induces either compressive or tensile strain in $\mathrm{MgO}$ corresponding to different polarities of the field [23]. Based on these results, a general consensus achieved that the electric field control of magnetic anisotropy is a subtle effect that might be driven by collective mechanisms, speculating that those involving structural modifications probably dominate the charge contribution. So far, theory has addressed the electric field control of magnetic anisotropy via inducedcharge changes [18-20] since ionic relaxation mechanisms are not well described by density functional theory under electric field. Thorough understanding of charge-mediated electric field control of PMA at ferromagnet/metal-oxide interfaces is still needed. It was shown that the strong PMA observed in $(\mathrm{Co}) \mathrm{Fe} / \mathrm{MgO}(\mathrm{AlOx})$ primarily originates from strong hybridizations between the interfacial $(\mathrm{Co}) \mathrm{Fe}-3 d$ and the $\mathrm{O}-2 p$ orbitals combined with spin-orbit coupling (SOC) [24]. Furthermore, a deeper on-site projected analysis also showed that the anisotropy energy is not only localized at the first monolayer, but also propagates into the bulk with an attenuating oscillatory behavior which depends on the orbital character of the contributing states and the interfacial conditions [25]. Thus, further understanding of the electric field impact on the PMA is anticipated.

In this article, we introduce an approach to investigate in detail the charge-driven mechanism underlying the electric field control on the PMA of $\mathrm{Fe} / \mathrm{MgO}$ interfaces from first principles. Namely, we elucidate this effect in relation to the intrinsic electric dipole at the $\mathrm{Fe} / \mathrm{MgO}$ interface, which has been observed and quantified. The increase (decrease) of PMA under an electric field is associated with a decrease (increase) of the interfacial electric dipole field which spontaneously exists even without application of any electrical field. Moreover, our on-site projected PMA analysis elucidates that even if the electric field is screened on a very short Thomas-Fermi length (1-2 $⿱$ ) in the metallic electrode, its influence extends beyond the interfacial Fe layer. In particular, we show that the main contribution to the PMA variation arises from the second Fe layer, a behavior which is understood in view of orbital hybridizations. 
Our first-principles calculations are based on the projectoraugmented wave (PAW) method [26] as implemented in the VASP package [27-29] using the generalized gradient approximation [30] and including spin-orbit coupling. A kinetic energy cutoff of $500 \mathrm{eV}$ has been used for the plane-wave basis set and a $25 \times 25 \times 1 k$-point mesh to sample the first Brillouin zone. The electric field, applied perpendicular to the interface, is introduced as a dipole layer placed in the vacuum region of the supercell, as proposed by the dipole layer method [31]. The orbital and layer-resolved magnetic anisotropies are evaluated following Refs. [25,32]. The supercell comprises five $\mathrm{Fe}$ monolayers (ML) sandwiched between two $5 \mathrm{ML}$ of $\mathrm{MgO}$, followed by a vacuum layer. The in-plane lattice constant was fixed to that of $\mathrm{Fe}$ (i.e., $a=2.87 \AA$ ), while the structure was relaxed in the absence of electric field until the forces became lower than $1 \mathrm{meV} / \AA$. We chose this symmetric structure since it provides the opportunity to compare the required physical properties of two different $\mathrm{Fe} / \mathrm{MgO}$ interfaces simultaneously in one calculation. On the contrary, by using an asymmetric geometry, e.g., $\mathrm{Fe} / \mathrm{MgO}$, the dipole moment arising from the asymmetric surface terminations is in principle corrected by the dipole layer method in Ref. [31]. In this asymmetric geometry, when an additional dipole opposite to the dipole correction is introduced within the vacuum region, the impact of the electric field itself would be difficult to quantify.

So far, the magnetoelectric effect at the $\mathrm{Fe} / \mathrm{MgO}$ interface has been discussed as a function of the electric field within the $\mathrm{MgO}$, in consistency with experiments where a potential difference is applied between two metal electrodes across the dielectric. However, a thorough analysis on the microscopic level of electric-field-induced changes in the electrostatic potential is needed to clarify the discussed phenomenon. Figure 1 shows the change in the planar-averaged electrostatic potential across the supercell when an electric field of $-2 \mathrm{~V} / \mathrm{nm}$ is applied. It is clear that the potential drop occurring in the $\mathrm{MgO}$ extends towards the Fe interface, whereas it is

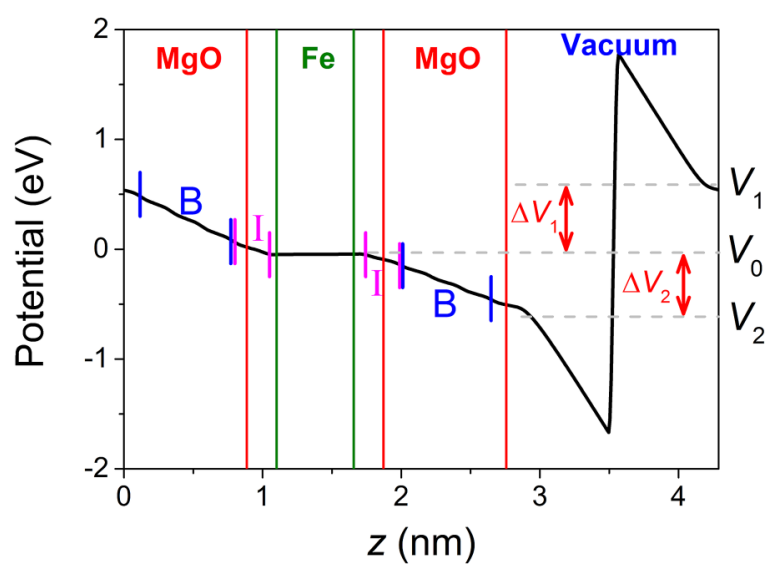

FIG. 1. Induced changes in the electrostatic potential under an electric field of $-2 \mathrm{~V} / \mathrm{nm}$ applied in the vacuum region of a $\mathrm{MgO} / \mathrm{Fe} / \mathrm{MgO}$ supercell. The vertical red and green lines represent the boundaries between $\mathrm{MgO}$ and Fe layers, respectively. Two different regions of the potential gradient are identified in the bulk of $\mathrm{MgO}$ and at the Fe interface, designated by $\mathrm{B}$ and $\mathrm{I}$, respectively. $\Delta V_{1}$ and $\Delta V_{2}$ designate the potential difference across the $\mathrm{MgO}$ where the reference potential $V_{0}$ is set as the Fe potential.

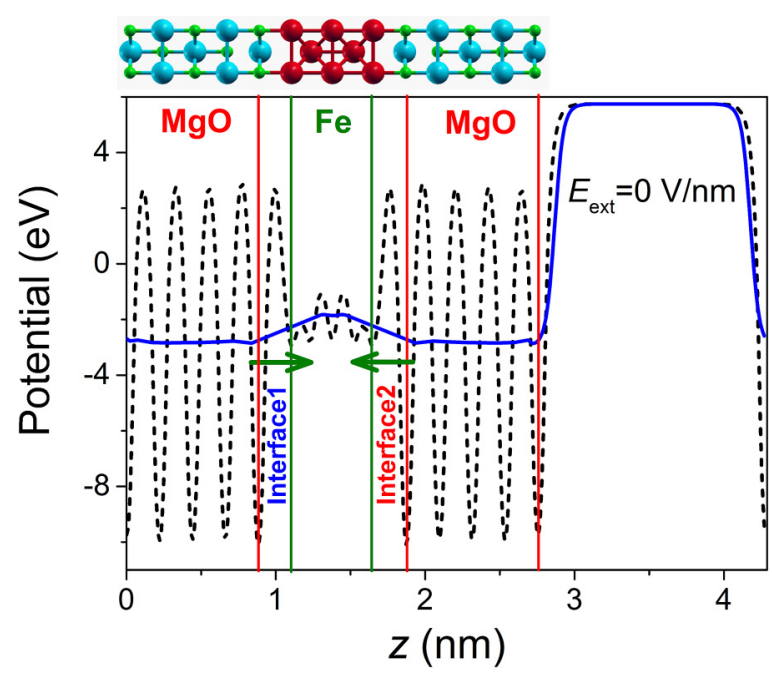

FIG. 2. Planar-averaged electrostatic potential (dashed line) and its macroscopic average (solid line) across a $\mathrm{MgO} / \mathrm{Fe} / \mathrm{MgO}$ supercell (top panel) calculated in the absence of electric field. The changes in the potential at both interfaces are the signature of the presence of an electric dipole whose direction is shown by the arrows.

screened in the bulk of Fe. Interestingly, one can distinguish between two different regions of the potential gradient: While it is steep in the bulk of $\mathrm{MgO}$, denoted by region $\mathrm{B}$ in Fig. 1, with a corresponding slope of about $0.61 \mathrm{~V} / \mathrm{nm}$, it becomes smoother at the interface $\sim 0.37 \mathrm{~V} / \mathrm{nm}$ (region I). This observation raises the query on a more detailed description of the phenomenon taking place at the interface, an objective which is accessible through macroscopic averaging on the atomic cell scale of the electrostatic potential. This allows filtering out the bulk oscillations in the potential using the atomic interlayer distance, thus highlighting the change of the potential at the interface [33]. Initially, when no electric field is applied, the planar-averaged and the macroscopically averaged electrostatic potentials are shown in Fig. 2. The nonuniformity of the microscopically averaged potential indicates the presence of two opposite dipoles at the $\mathrm{Fe} / \mathrm{MgO}$ and $\mathrm{MgO} / \mathrm{Fe}$ interfaces and allows us to define an intrinsic interfacial dipole which obviously comprises $1 \mathrm{ML}$ of $\mathrm{MgO}$ and $2 \mathrm{ML}$ of Fe. Moreover, we evaluate the field associated with this interfacial dipole from the gradient of the potential at each interface and find $E_{\text {dip }}=2.2 \mathrm{~V} / \mathrm{nm}$ pointing towards the $\mathrm{Fe}$ layer. Of note, the existence of this dipole in principle could lead to an observation of the Rashba effect at $\mathrm{Fe} / \mathrm{MgO}$ and other similar structures. This is out of the scope of the current work and will be addressed separately.

To assess the effect of an external electric field on the $\mathrm{Fe} / \mathrm{MgO}$ interfacial dipole, we perform a macroscopic averaging of the electrostatic potential of the same supercell under an external electric field of $-2 \mathrm{~V} / \mathrm{nm}$. We find that the magnitude of $E_{\text {dip }}$ decreases by $0.2 \mathrm{~V} / \mathrm{nm}$ on the first interface while it increases by the same value on the second. This behavior is interpreted in terms of changes in the interfacial charge population induced by the applied field. A charge depletion $\Delta n=-0.01 e^{-}$takes place at the first interface, yielding a decrease in $E_{\text {dip }}$. Conversely, a charge accumulation appears on the second interface, resulting in an increase in $E_{\text {dip }}$. 


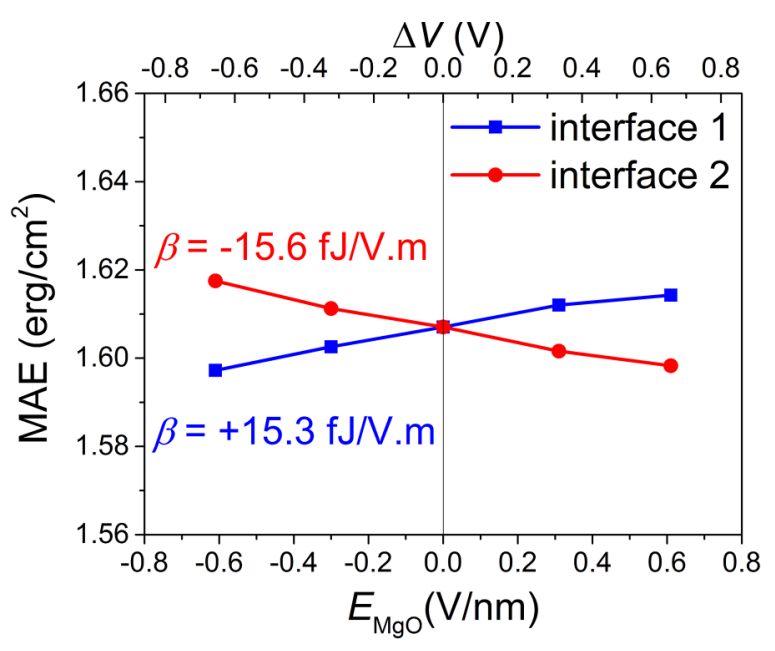

FIG. 3. The variation of the total magnetic anisotropy energy (MAE) per interface, comprising the first and second Fe monolayers, as a function of the electric field in the bulk of $\mathrm{MgO}\left(E_{\mathrm{MgO}}\right)$ calculated by applying an electric field ranging from -2 to $2 \mathrm{~V} / \mathrm{nm}$ in the vacuum region of the $\mathrm{MgO} / \mathrm{Fe} / \mathrm{MgO}$ supercell. The data points are linearly fitted to evaluate the slope $\beta$ associated with the PMA response to the electric field.

In the following, we discuss the changes in the PMA of $\mathrm{Fe} / \mathrm{MgO}$ induced by an electric field in view of the interfacial dipole. The variation of the PMA per interface, comprising the first and second Fe layers, as a function of both the electric field in the bulk of $\mathrm{MgO}\left(E_{\mathrm{MgO}}\right)$ and the potential difference across $\mathrm{MgO}, \Delta V=\Delta V_{1}=-\Delta V_{2}$, is shown in Fig. 3. It is noteworthy that the values of $\Delta V$ correspond to the potential difference calculated as demonstrated in Fig. 1, such that the reference potential is chosen to be the $\mathrm{Fe}$ potential, which gives direct insight into what is measured experimentally. The behavior of the two interfaces infers that the decrease (increase) of $E_{\text {dip }}$, arising from charge depletion (accumulation), is associated with an increase (decrease) of the PMA correspondingly. This correlation between the interfacial $\mathrm{Fe} / \mathrm{MgO}$ dipole and the PMA exists even in the absence of an applied electric field. In fact, one can modulate the dipole amplitude by imposing some structural modifications. For instance, if the equilibrium position of the $\mathrm{Mg}$ interfacial atom is forced to be shifted off the $\mathrm{O}$ plane toward $\mathrm{Fe}$ by a quantity $\Delta Z$, then $E_{\text {dip }}$ and correspondingly the total PMA of the supercell, comprising two interfaces, varies as shown in Fig. 4, in agreement with the correlation identified above.

To get more insight into the electric field effect on the PMA of $\mathrm{Fe} / \mathrm{MgO}$ interfaces, we evaluate the layer-resolved contributions to the total anisotropy value. Figure 5 shows the on-site projected magnetic anisotropy energy as a function of $E_{\mathrm{MgO}}$. The effect of the electric field on the PMA is quantified by the slope $\beta$. Interestingly, we observe that the PMA of the 1st ML of Fe exhibits a nonlinear behavior that is an even function of the polarity of the electric field with $\beta=-3 \mathrm{fJ} /(\mathrm{V} \mathrm{m})$. On the other hand, the linear behavior of the PMA of the $2 \mathrm{ML}$, with a corresponding $\beta=+14.8$ and $-15.2 \mathrm{fJ} /(\mathrm{V} \mathrm{m})$ for interfaces 1 and 2 , respectively, carries the main contribution to the behavior of the total PMA in response to electric field, whose slope is $\beta=+15.3 \mathrm{fJ} /(\mathrm{V} \mathrm{m})$

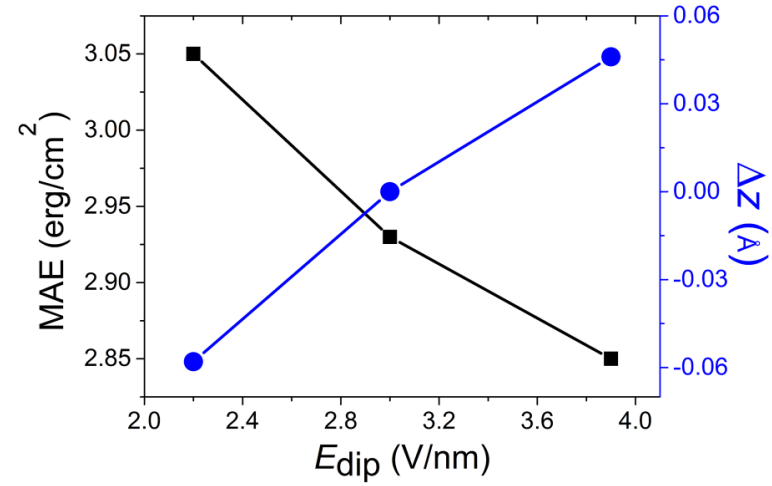

FIG. 4. The variation of the total MAE of the supercell, comprising two interfaces, as a function of the interfacial electric dipole $E_{\text {dip }}$ which is modulated by the shift of the $\mathrm{Mg}$ position with respect to the $\mathrm{O}$ plane $\Delta Z$.

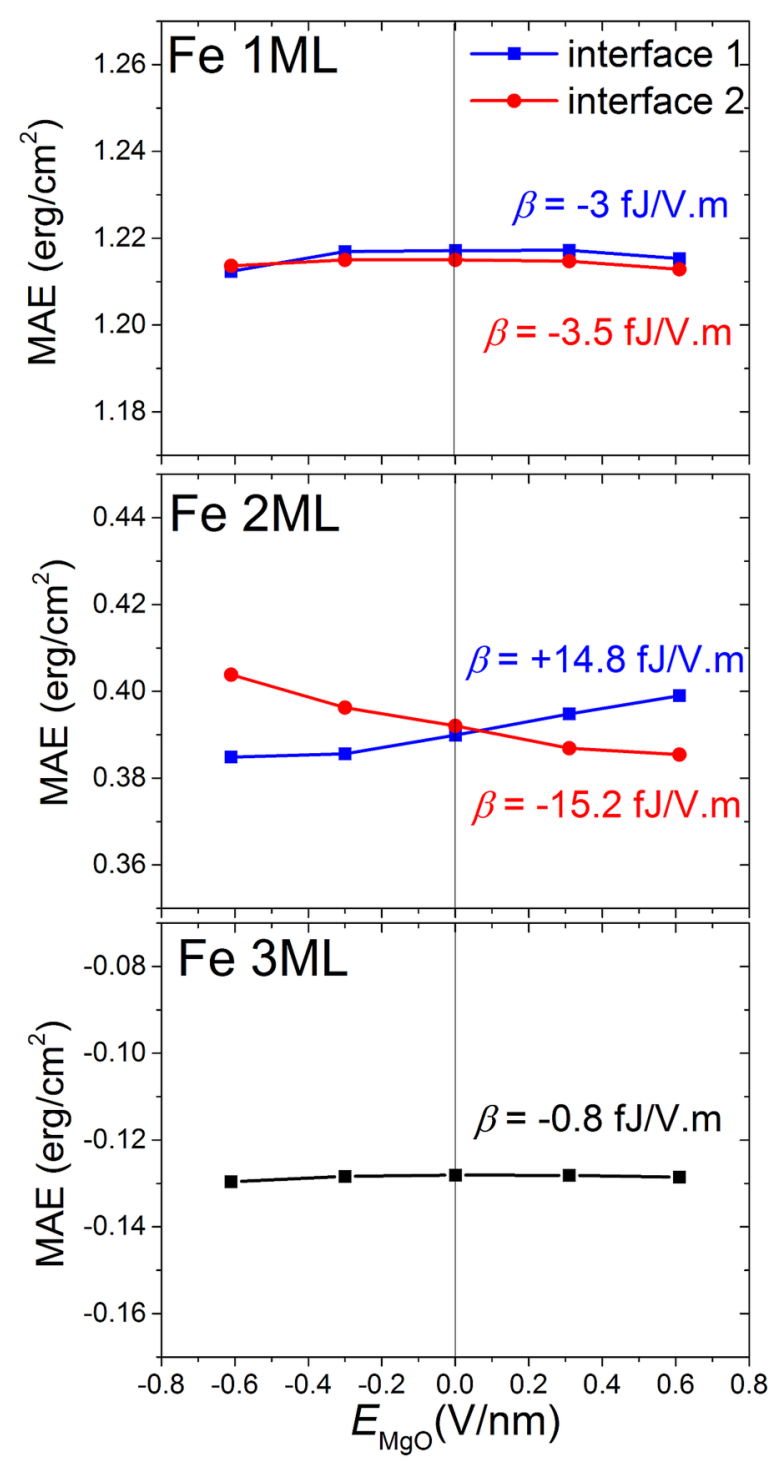

FIG. 5. On-site projected MAE as a function of $E_{\mathrm{MgO}}$ calculated for the first, second, and third Fe monolayers. The slope $\beta$ of the MAE variation is evaluated and displayed for each ML. 
(a)

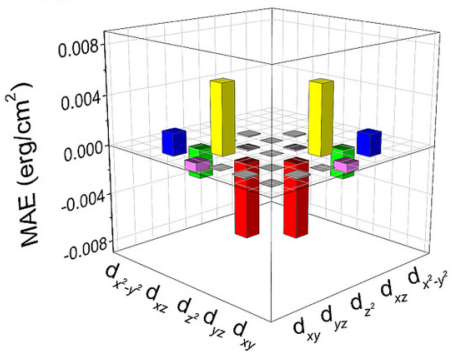

Fe-1ML

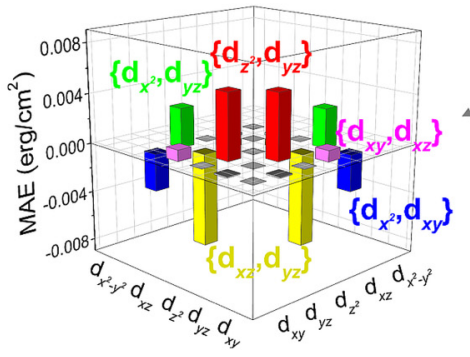

(c)

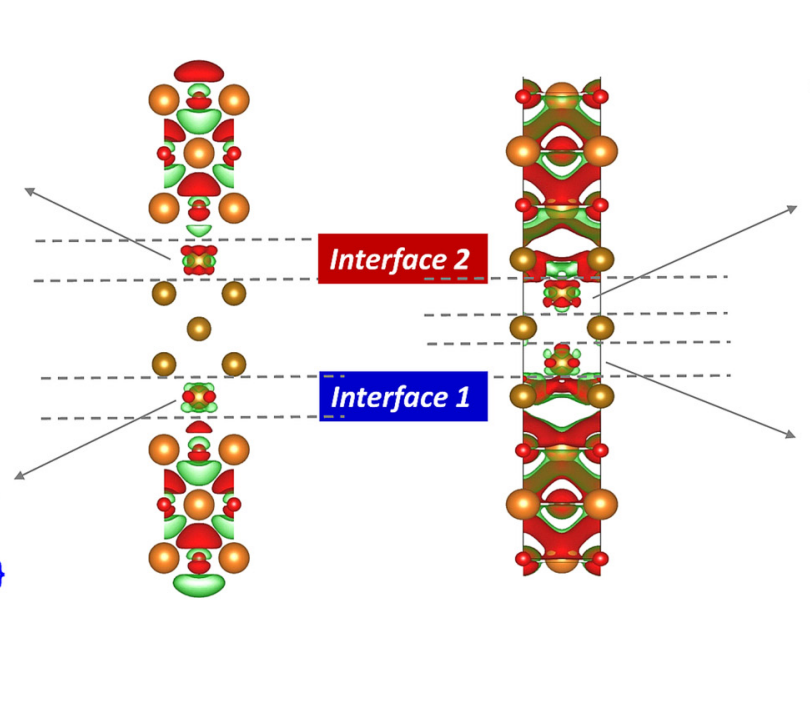

Fe-2ML

(b)

FIG. 6. (a), (b) The induced changes in the magnetic anisotropy of the orbital hybridization calculated for 1st and 2nd ML of Fe, respectively, when an electric field of $-2 \mathrm{~V} / \mathrm{nm}$ is applied. The corresponding changes in the charge distribution plotted using an isovalue of $\Delta n=10^{-4} e \AA^{-3}$ for $1 \mathrm{ML}$ (c) and $\Delta n=10^{-5} e \AA^{-3}$ for $2 \mathrm{ML}$ (d) of Fe. The red (green) region indicates an accumulation (depletion) of electrons.

and $-15.6 \mathrm{fJ} /(\mathrm{V} \mathrm{m})$, as shown in Fig. 3. In line with the previously demonstrated aspect of the PMA in $\mathrm{Fe} / \mathrm{MgO}$ [25], our finding indicates that the electric field impact on the PMA is not localized at the interface, but it rather extends to the $2 \mathrm{ML}$ which even dominates the $1 \mathrm{ML}$ contribution.

The dominant contribution of the $2 \mathrm{ML}$ of $\mathrm{Fe}$ to the total PMA variation as a function of electric field can be interpreted in view of the orbital hybridization. The changes in the magnetic anisotropy of the orbital hybridization induced by an electric field of $-2 \mathrm{~V} / \mathrm{nm}$ are shown in Fig. 6 for both 1st and 2 nd ML of Fe on each interface. We adopt such threedimensional plots, rather than the integrated two-dimensional ones, in order to further visualize every $d$-orbital hybridization contributions which are hidden in two-dimensional plots, namely, in a situation where the same $d$ orbital may give positive or negative electric field controlled PMA contributions depending on the other $d$ orbital it is hybridized with. Furthermore, we think that this is more suitable also from a fundamental point of view since these individual hybridization contributions represent matrix elements of the spin-orbit part of the Hamiltonian. In other words, it is a more constructive way to give the mechanisms of the effect on the smallest possible microscopic scale. Moreover, the induced changes in the charge distribution, shown in Fig. 6, demonstrate that the screening of the electric field occurs beyond the $2 \mathrm{ML}$ of $\mathrm{Fe}$. Although the induced charge on the $\mathrm{Fe}-3 d$ orbitals of the $2 \mathrm{ML}$ is much less compared to that of the $1 \mathrm{ML}$, this is rendered differently in terms of the anisotropy of the orbitals. For instance, considering the $1 \mathrm{ML}$ of $\mathrm{Fe}$ in interface 1 , some orbitals, specifically the $\left(d_{z^{2}-r^{2}}, d_{y z}\right)$ and $\left(d_{x^{2}-y^{2}}, d_{y z}\right)$, are contributing an increase of the anisotropy while others contribute a decrease of PMA, such as $\left(d_{x z}, d_{y z}\right)$ and $\left(d_{x^{2}-y^{2}}, d_{x y}\right)$. Those counterbalanced contributions result in a negligible overall effect of the electric field on the PMA of the first Fe layer. On the other hand, one can clearly see that all the orbitals of the 2 ML contribute an increase of the anisotropy, giving rise to a substantial change of the second layer's PMA, which accounts for most of the overall response of the $\mathrm{Fe} / \mathrm{MgO}$ interface to an applied electric field.

The aforementioned results on $\mathrm{MgO}(5 \mathrm{ML}) / \mathrm{Fe}(5 \mathrm{ML}) /$ $\mathrm{MgO}(5 \mathrm{ML})$ have been further verified for different thicknesses of $\mathrm{Fe}$ and $\mathrm{MgO}$. We were able to reproduce the same behavior of the layer-resolved PMA as a function of electric field, in particular, the dominating contribution of the second Fe ML. The average value calculated for the slope $\beta$ per interface is $15 \pm 2.5 \mathrm{fJ} /(\mathrm{V} \mathrm{m})$. Consequently, we believe that this microscopic mechanism that is responsible for the electric field control of PMA in $\mathrm{Fe} / \mathrm{MgO}$ interfaces can be generalized to $\mathrm{MgO} / \mathrm{Fe} /$ metal heterostructures, regardless of the mechanism that might take place at the Fe/metal interface. In fact, it has been recently demonstrated that the interfacial $\mathrm{Fe} / \mathrm{MgO}$ PMA is almost unaffected by the other $\mathrm{Fe} /$ metal interface in such heterostructures comprising several monolayers [34].

In summary, we have presented a microscopic description of the charge-mediated electric field effect on the PMA of $\mathrm{Fe} / \mathrm{MgO}$ interfaces. We have correlated this effect to the intrinsic dipole at the $\mathrm{Fe} / \mathrm{MgO}$ interface. Furthermore, we verified the firm relation between the interfacial dipole and the PMA even in the absence of an applied electric field, highlighting that an increase of dipole amplitude leads to a decrease in PMA. The on-site and orbital projected contributions to the PMA as a function of electric field were also evaluated and, interestingly, it turns out that the second Fe monolayer dictates the overall variation of the PMA of $\mathrm{Fe} / \mathrm{MgO}$ in response to an applied electric field. Finally, we anticipate that the interfacial intrinsic dipole could lead to the observation of a Rashba 
effect and related spin orbitronic phenomena in $\mathrm{Fe} / \mathrm{MgO}$ and other transition metal/oxide interfaces, which will be reported elsewhere.
We acknowledge P. Lukashev and E. Tsymbal for fruitful discussions. This work was partly supported by the ERC Advanced 276 Grant Project MAGICAL No. 669204.
[1] J. C. Slonczewski, J. Magn. Magn. Mater. 159, L1 (1996).

[2] L. Berger, Phys. Rev. B 54, 9353 (1996).

[3] J. Z. Sun, J. Magn. Magn. Mater. 202, 157 (1999).

[4] E. B. Myers, D. C. Ralph, J. A. Katine, R. N. Louie, and R. A. Buhrman, Science 285, 867 (1999).

[5] A. Brataas, A. D. Kent, and H. Ohno, Nat. Mater. 11, 372 (2012).

[6] S. Ikeda, K. Miura, H. Yamamoto, K. Mizunuma, H. D. Gan, M. Endo, S. Kanai, J. Hayakawa, F. Matsukura, and H. Ohno, Nat. Mater. 9, 721 (2010).

[7] C. Chappert, A. Fert, and F. N. Van Dau, Nat. Mater. 6, 813 (2007).

[8] K. Mizunuma, S. Ikeda, J. H. Park, H. Yamamoto, H. Gan, K. Miura, H. Hasegawa, J. Hayakawa, F. Matsukura, and H. Ohno, Appl. Phys. Lett. 95, 232516 (2009).

[9] C. Ducruet, B. Carvello, B. Rodmacq, S. Auffret, G. Gaudin, and B. Dieny, J. Appl. Phys. 103, 07 A918 (2008).

[10] B. Dieny and O. Redon, U.S. Patent No. 6,950,335 B2 (16 November 2001).

[11] G. Kim, Y. Sakuraba, M. Oogane, Y. Ando, and T. Miyazaki, Appl. Phys. Lett. 92, 172502 (2008).

[12] D. Chiba, M. Yamanouchi, F. Matsukura, and H. Ohno, Science 301, 943 (2003).

[13] M. Weisheit, S. Fähler, A. Marty, Y. Souche, C. Poinsignon, and D. Givord, Science 315, 349 (2007).

[14] T. Maruyama, Y. Shiota, T. Nozaki, K. Ohta, N. Toda, M. Mizuguchi, A. A. Tulapurkar, T. Shinjo, M. Shiraishi, S. Mizukami, Y. Ando, and Y. Suzuki, Nat. Nanotechnol. 4, 158 (2009).

[15] T. Nozaki, Y. Shiota, M. Shiraishi, T. Shinjo, and Y. Suzuki, Appl. Phys. Lett. 96, 022506 (2010).

[16] W. G. Wang, M. Li, S. Hageman, and C. L. Chien, Nat. Mater. 11, 64 (2012).

[17] A. Rajanikanth, T. Hauet, F. Montaigne, S. Mangin, and S. Andrieu, Appl. Phys. Lett. 103, 062402 (2013).
[18] C. G. Duan, J. P. Velev, R. F. Sabirianov, Z. Zhu, J. Chu, S. S. Jaswal, and E. Y. Tsymbal, Phys. Rev. Lett. 101, 137201 (2008).

[19] M. K. Niranjan, C. G. Duan, S. S. Jaswal, and E. Y. Tsymbal, Appl. Phys. Lett. 96, 222504 (2010).

[20] K. Nakamura, T. Akiyama, T. Ito, M. Weinert, and A. J. Freeman, J. Magn. 16, 161 (2011).

[21] F. Bonell, Y. T. Takahashi, D. D. Lam, S. Yoshida, Y. Shiota, S. Miwa, T. Nakamura, and Y. Suzuki, Appl. Phys. Lett. 102, 152401 (2013).

[22] U. Bauer, L. Yao, A. J. Tan, P. Agrawal, S. Emori, H. L. Tuller, S. V. Dijken, and G. S. D. Beach, Nat. Mater. 14, 174 (2015).

[23] V. B. Naik, H. Meng, J. X. Xiao, R. S. Liu, A. Kumar, K. Y. Zeng, P. Luo, and S. Yap, Appl. Phys. Lett. 105, 052403 (2014); G. Q. Yu, Z. X. Wang, M. Abolfath-Beygi, C. He, X. Li, K. L. Wong, P. Nordeen, H. Wu, G. P. Carman, X. F. Han, I. A. Alhomoudi, P. Khalili Amiri, and K. L. Wang, Appl. Phys. Lett. 106, 072402 (2015).

[24] H. X. Yang, M. Chshiev, B. Dieny, J. H. Lee, A. Manchon, and K. H. Shin, Phys. Rev. B 84, 054401 (2011).

[25] A. Hallal, H. X. Yang, B. Dieny, and M. Chshiev, Phys. Rev. B 88, 184423 (2013).

[26] P. E. Blöchl, Phys. Rev. B 50, 17953 (1994).

[27] G. Kresse and J. Hafner, Phys. Rev. B 47, 558 (1993).

[28] G. Kresse and J. Furthmuller, Phys. Rev. B 54, 11169 (1996).

[29] G. Kresse and J. Furthmuller, Comput. Mater. Sci. 6, 15 (1996).

[30] J. P. Perdew, K. Burke, and M. Ernzerhof, Phys. Rev. Lett. 77, 3865 (1996).

[31] J. Neugebauer and M. Scheffler, Phys. Rev. B 46, 16067 (1992).

[32] A. Hallal, B. Dieny, and M. Chshiev, Phys. Rev. B 90, 064422 (2014).

[33] D. M. Bylander and L. Kleinman, Phys. Rev. B 36, 3229 (1987).

[34] S. Peng, M. Wang, H. Yang, L. Zeng, J. Nan, J. Zhou, Y. Zhang, A. Hallal, M. Chshiev, K. L. Wang, Q. Zhang, and W. Zhao, Sci. Rep. 5, 18173 (2015). 